\title{
La teoría del capital humano, fundamento del programa Beca 18
}

\section{RESUMEN}

El objetivo de esta investigación es examinar cómo la teoría del capital humano se constituye en el fundamento principal del programa Beca 18. Aunque su formulación incluye enfoques como la inclusión y la exclusión social, enfoques como el desarrollo con equidad, teorías tales como desarrollo humano, la teoría del capital humano es la que se destaca como el sustento en que se apoya y organiza. Un examen de las expectativas del programa, de sus indicadores, del tipo de financiamiento, así como de los filtros que establece en su ejecución, reafirma nuestra conclusión. La influencia determinante de esta teoría en el diseño del programa permite descubrir su sesgo economicista, cuyo objetivo es promover en el grupo de beneficiarios la búsqueda de un ingreso económico por encima de la línea de pobreza, pero no su desarrollo multilateral.

Palabras clave: Capital humano; capital; pobreza; desarrollo; productividad.

\section{Human capital theory, the foundation of the Beca 18 program}

\section{ABSTRACT}

This research objective is to examine how the human capital theory establishes as the main foundation of the «Beca 18» program. Although its formulation includes approaches such as social inclusion and exclusion, equity and human development; the human capital theory stands out as a foundation on which it is supported and organized. By examining the program's expectations, indicators, funding types, filters arranged in execution, our conclusion is reaffirmed. The determinant influence of this theory in the program's design makes possible to uncover its economistic bias, whose objective is to promote in the beneficiary group the search for an economic income above the poverty line, but not its multilateral development.

KeYworDs: Human capital; capital; poverty; development; productivity. 


\section{Introducción}

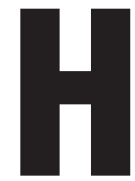
acia fines de los 50, y durante la década de los 60 del siglo pasado, se formula en sus líneas más importantes la teoría del capital humano. Tuvo, entonces, gran influencia en el mundo de la educación, sobre todo en los EE.UU. Sus mentores más conocidos fueron Theodore Schultz, Gary Becker y Jacob Mincer, entre otros.

A fines de siglo xx e inicios del xxI, el mundo laboral sufre considerables cambios. La globalización y la economía de mercado, impulsadas por el Consenso de Washington, abren una situación nueva, donde los empleos para trabajadores «dotados de mayores conocimientos» resultan ser de alta importancia para el desarrollo económico. Avanza el conocimiento científico tecnológico, y se exige el avance de la capacidad y los conocimientos de los trabajadores.

La teoría del capital humano logra en este contexto un gran resurgimiento; en países como el caso del Perú, la asumen como guía de la relación que debe existir entre educación y producción, y como un fundamento de la política oficial seguida contra la pobreza.

Con la formulación e implementación de Programas como Beca 18 se apoya el acceso, la permanencia y graduación en el nivel superior de estudios de jóvenes que tengan rendimiento académico sobresaliente y que, a su vez, sean parte de la población en condición de pobreza o pobreza extrema. En el Perú 38\% de los jóvenes declara que no asistía a una institución de educación superior por problemas económicos; y el 25\%, por razones de trabajo.

Para Beca 18, eliminar la pobreza es invertir en educación; específicamente, es dotar a los jóvenes de mayores y mejores conocimientos, desarrollar sus capacidades, sus habilidades y destrezas en carreras que tengan alta rentabilidad, lo cual les permitirá desarrollarse como fuerza de trabajo calificada y obtener mayor ingreso a futuro, obtener prosperidad individual y salir de la pobreza.

El programa ha seleccionado las carreras que tienen mayor rentabilidad y que son consideradas necesarias a la actual estructura productiva peruana. Les otorga a los becarios el apoyo económico y la asistencia académica, siempre que esta última esté dentro de los límites de rentabilidad de la inversión. Los beneficiarios al finalizar los estudios se comprometen a laborar en el país durante tres años, especialmente en su zona de procedencia.

En la concepción del programa Beca 18 concurren teorías como las de la exclusión e inclusión social, difundidas por la OIT; enfoques como el del desarrollo con equidad; teorías como las del desarrollo humano; sin embargo, la que configura y ordena su fundamento es la del capital humano, hecho que se produce en plena concordancia con la política del Consenso de Washington, implementada en estos 26 ańos. Esta teoría adquiere una presencia amplia y sistematizada en este programa, con proyección de constituirse como fundamento de la política a seguir en la educación superior. Trabajos como los de Yamada (2007, 2010, 2012, 2016), Rivera (2012), Castro $(2004,2010)$ han sistematizado en el Perú una versión de ella.

En este artículo vamos a desarrollar, en primer lugar, una síntesis de las tesis más importantes de la teoría del capital humano, luego presentaremos el diseño del programa Beca 18 y, finalmente, un comentario sobre ambos.

Para el presente trabajo se ha seleccionado los textos representativos que exponen y sustentan la teoría educativa del capital humano, el más importante de ellos es el de Gary Becker. Luego, para el caso del Perú, están los trabajos del equipo de economistas de la Universidad del Pacífico que lideran su aplicación. Hemos procedido a identificar las tesis centrales, incluyendo las que plantean los teóricos de la OCDE, quienes han logrado un desarrollo específico con la teoría de las competencias y la prueba PISA. Se ha construido así un breve cuerpo con las tesis centrales de esta teoría, modelo teórico que da perfil a este punto de vista. Luego, de los documentos oficiales del Programa Beca 18, publicados por pronabec (2012, $2013,2014)$ y el MED $(2011,2012,2014)$ hemos extraído la fundamentación del programa, habiendo hallado las coincidencias centrales entre ambos.

A continuación, a partir de las tesis de Marx, expuestas en El capital, hemos realizado la consideración de los resultados. Este trabajo es fundamentalmente de análisis de fuentes escritas. Expuestos los resultados y realizada la apreciación correspondiente, procedemos a señalar nuestras conclusiones. El trabajo se definió como exploratorio y fundamentalmente cualitativo. 


\section{La teoría del capital humano}

Desde esta posición se sostiene que las personas invierten en sí mismas buena parte de sus recursos (dinero o tiempo) con la esperanza de obtener beneficios futuros. Tres investigadores resultan fundamentales en su definición y desarrollo como teoría, así como en su aplicación al análisis concreto: Theodore Schultz, Gary Becker y Jacob Mincer.

\subsection{Las tesis de T. Schultz}

Schultz entiende la adquisición de capital humano como un proceso por el cual se accede a "conocimientos y habilidades» (1999, p. 85), encaminados a incrementar la productividad y a producir ganancia. Por esta razón, le correspondería la calificación de capital, pero con características distintas a las del capital físico. Para obtener estos "conocimientos y habilidades» se requiere de una inversión ideada hacia este propósito específico.

Sostiene que: "Por estos medios, y otros similares, puede aumentar mucho la calidad del esfuerzo humano" (1999, p. 85). Afirma que el incremento de los gastos para mejorar estas capacidades permite que la productividad del esfuerzo humano (trabajo) proporcione una tasa positiva de rendimiento (1999, p. 88). Schultz (1999) quiere demostrar que «esas inversiones en capital humano pueden explicar la mayor parte del enorme aumento de las ganancias reales por trabajador» (p. 85). A su opinión, le «parece elemental la deducción de que las diferencias en la cantidad de inversión humana puedan explicar las diferencias en ingresos» (1999, p. 88).

\subsection{A estas tesis expuestas se añaden las contribuciones de Gary Becker}

Becker sostiene sus propuestas más importantes en su libro El capital humano. En él examina los efectos que la inversión en educación y formación tienen sobre las retribuciones y sobre las tasas de rendimiento.

Expone la naturaleza del capital humano en los siguientes términos:

Voy a hablar de otro tipo de capital. La educación, el cuidado de la salud, un curso de capacitación en computación, las lecciones sobre virtudes como la puntualidad y la honestidad constituyen también un capital, en el sentido que mejoran la salud, aumentan las ganancias, o añaden un mejor entendimiento de la literatura durante gran parte de su vida. Así, es totalmente válido y concordante con el concepto tradicional de capital sostener que los gastos en educación, salud, capacitación, etc. son inversiones de capital. Sin embargo, estos producen capitales humanos, no bienes físicos o financieros, porque no se puede separar a una persona de sus conocimientos, de sus habilidades, de su salud o de sus valores como sí es posible retirar bienes físicos y financieros de una persona así los esté usando (1983, pp. 15-16).

La salud física y psíquica es considerada por esta línea teórica como una modalidad de capital humano. En el decir de Becker (1983), la capacidad física y la estabilidad emocional son "como un determinante fundamental de las retribuciones en todas partes del mundo" (p. 54).

Becker: la inversión en capital humano y sus efectos sobre las retribuciones. Las personas invierten parte de sus recursos (ya sea tiempo o dinero) en sí mismas: en estudio, en formación, en adquirir experiencia. Es una inversión hecha para adquirir conocimientos, habilidades, capacidades y otros recursos que influirán en la vida económica y en sus ingresos futuros, constituyen un elemento clave de la productividad, del crecimiento económico y del incremento del ingreso (Becker, 1983, pp. 26-28).

Para la teoría del capital humano, los estudiantes o trabajadores elevan su productividad y sus ingresos adquiriendo algunas calificaciones y perfeccionando otras. Los costos para lograr este incremento incluyen el valor que se asigna tanto al tiempo utilizado como al esfuerzo desplegado; incluyen, asimismo, la «enseñanza» que los profesores o instructores imparten, así como los equipos y materiales que se utilizan ${ }^{1}$. Estos costos pueden ser, a su vez, directos (gastos en matrícula, pasajes, equipos, libros, etc.) e indirectos (el ingreso que pudimos lograr en el tiempo que utilizamos para adquirir estos conocimientos y capacidades).

1 Becker (1983) aclara que: «estas partidas son costes, porque de no haberse utilizado para incrementar la producción futura podrían haber sido utilizadas para obtener producto en el presente. El gasto y el tiempo dedicados al aprendizaje dependen, en parte, del tipo de formación; se gasta más y durante un período más largo de tiempo, en un residente médico que en un mecánico por ejemplo» (p.29) 
Becker: la inversión en capital humano y sus efectos sobre las tasas de rendimiento. Si una persona gana ochocientos soles y otra cuatro mil, esta distribución de la renta, diría, es explicable por la diferencia de capital humano que existe entre ambas. Por eso, dice Becker (1983), poner "el énfasis en la importancia del capital humano no solo ayuda a explicar las diferencias entre retribuciones a lo largo del tiempo, así como entre áreas geográficas, sino también entre personas o familias dentro de una misma unidad geográfica» (p. 99).

Su teoría también examina los costes de inversión en capital humano en relación con el factor edad: la cantidad de tiempo invertido en capital humano tendería a disminuir con la mayor edad (1983, p. 79), esta supone un costo mayor, pues durante el tiempo que estudia o se capacita se deja de percibir montos de ingresos que en los trabajadores de más edad se suponen más elevados; a esto se agrega que el tiempo que gozaría de los mejores ingresos sería menor por la edad avanzada y la esperanza de vida existente; finalmente, agrega, resultará insuficiente el tiempo para recuperar la inversión. De aquí concluye que, a mayor edad, si bien los beneficios aumentan, lo hacen a una tasa decreciente, y se concluye en lo poco rentable que resultaría invertir en personas adultas (1983, pp. 71-86),

La tasa de retorno y la magnitud de las inversiones. El retorno ${ }^{2}$ de la inversión en capital humano resultaría ser el factor más importante para determinar cuánto invertir en capital humano. La rentabilidad de invertir en educación, y el impacto que provoca en los sueldos, salarios y otros ingresos económicos, resulta un punto clave de esta teoría, pues señala el beneficio de la inversión en este campo.

Si un joven invierte quince mil dólares en una maestría, y logra recuperar este mismo monto, debido a un incremento en su sueldo favorecido por estos estudios, podríamos decir que su beneficio o retorno fue cero. Para explicar la causa de estos problemas, Becker subraya, en el marco teórico formulado, la relación que existe entre los recursos destinados a lograr capital humano y la heterogeneidad de ingresos que puedan alcanzarse; examina, a su vez, las relaciones

2 Yamada, G. y Cárdenas, M (2017) lo identifican como el «...ingreso adicional que una persona recibe una vez insertada en el mercado laboral por cada ańo o nivel adicional de educación que invirtió en su juventud» (p. 53) esenciales que operan entre retribuciones, costos de inversión y tasas de rendimiento (1983, p. 59).

"Cada individuo, señala, produce su propio capital humano utilizando parte de su tiempo y de sus bienes en la asistencia a una 'escuela', en recibir formación en el trabajo, etc.». (1983, pp. 77-78). El retorno de esta inversión no es inmediato; forma parte de un proceso que comprende, entre otros, el momento de la inversión, la cual es progresiva, y el momento de la recepción de los beneficios, que es la fase final. Becker concluye que una inversión en capital humano es rentable si las «retribuciones netas», son mayores que los costos. Por eso diría que, antes de decidirse a estudiar, uno se debería preguntar cuánto de ingreso adicional me reportará estudiar la maestría de arte, por ejemplo, en relación con el ingreso que me correspondería por ser solo licenciado. La conveniencia estaría basada, según la teoría del capital humano, en el conocimiento de este dato.

La tasa de retorno influye, así, en la relación esencial que se da entre el sistema educativo y el mercado de trabajo. Si los conocimientos que se imparten en la carrera universitaria de contabilidad, por ejemplo, tuviesen mucha demanda en el mercado de trabajo, y si contasen con remuneraciones o sueldos muy altos, esto llevaría a que muchos estuviesen interesados en estos estudios; este impacto atraería a muchos postulantes y proveería de muchos egresados, hasta que ocurriese la reducción de la demanda.

¿Cuál sería el monto ideal a invertir por una persona que desea incrementar su capital humano? ¿Hasta cuánto es posible destinar? "Los individuos eligen su nivel óptimo de inversión en educación hasta el punto en que los costos y los beneficios correspondientes se igualan» (Yamada, 2007, p. 21).

El modelo de oferta y demanda. Becker examina cómo el capital humano relaciona oferta y demanda. Define y toma como base el concepto de «ingreso neto ${ }^{3}$ (1983, pp. 59-60 y 111-113) elabora un modelo de oferta y demanda con el cual se propone explicar por qué las personas, las familias, las instituciones invierten montos diferenciados de capital humano, o por qué las personas que invierten igual monto de capital humano pueden obtener ingresos diferentes.

3 Resultan de sumar los ingresos que se hubieran obtenido con cero inversiones en capital humano, más los ingresos que resultan de inversiones previas de capital humano, menos los costes de inversión en el capital humano que se está considerando. (1983: 111) 
Examina, entonces, a cada una de ellas:

La curva de la demanda. (1983, pp. 114-118) Becker examina aquí los efectos que ocasiona una mayor acumulación de capital humano en un mismo trabajador. Dada su condición de inseparable portador del capital humano, las limitaciones de las capacidades físicas en el ser humano también ejercen cierto impacto en la persona que realiza la inversión (1983, p.114). Tienden por eso a elevar los costos necesarios para producir una unidad monetaria de capital humano (183, p.114). La parte de su tiempo que emplea la persona en producir capital humano, es más costosa a medida que este inversor acumula mayor cantidad de ese capital (1983, p.113).

La curva de la oferta. Muestra el costo que tiene financiar una unidad adicional de capital humano (1983, pp. 118-119); por ejemplo, pasar del nivel de educación secundaria a un nivel de educación superior; o de licenciado a magíster o doctor. Considera la facilidad o dificultad para conseguir el financiamiento. En los estudios, es frecuente la medición en años. Los costes aumentan con la cantidad y tipo de inversión.

Becker sostiene que en estos casos: «Una decisión racional implica seleccionar una senda que maximice el valor actualizado de los 'beneficios', esto es, el valor actualizado de la diferencia entre ingresos y costos» (1983, p. 119).

La dinámica de la oferta y la demanda explicaría así las diferencias en el capital humano invertido por las personas, según sea que varíe la oferta, la demanda o ambas (1983, p.119).

Yamada (2007) sostiene que: «el nivel de retorno de la educación y su evolución en el tiempo dependen de factores de oferta y demanda. En el caso de la oferta, se trata de la cantidad de profesionales y técnicos que egresan de las instituciones educativas e ingresan al mercado laboral. En el caso de la demanda se trata del aparato productivo de bienes y servicios que demanda la mano de obra calificada. Esta demanda se ve potencialmente afectada por el ritmo de crecimiento de la economía en su conjunto, por el modo de desarrollo vigente, por el grado de apertura de la economía, por el ritmo y sesgo del cambio tecnológico» (pp. 21-22).

Dos planteamientos de las distribuciones de las inversiones de capital humano y de las retribuciones de inversión del capital humano son sintetizados por Becker (1983) en los siguientes términos:

Planteamiento igualitario. En este caso las diferencias de inversión responden a diferentes posibilidades de financiamiento, lo cual proporciona a unos más oportunidades de acceder a capital humano que a otros (pp. 122-123).

Planteamiento «elitista». Para este caso "las inversiones y retribuciones reales diferirían principalmente en función de las diferencias de capacidades para beneficiarse de la inversión en capital humano. Algunos individuos son más aptos y constituyen una élite» (p. 125). Finalmente ambas teorías concurren para ofrecer una explicación más completa, dentro del campo de la teoría del capital humano.

\subsection{Jacob Mincer}

Otro punto clave en la teoría del capital humano lo constituye la famosa «ecuación de ingresos de Mincer». Con ella se busca explicar el monto salarial que se obtiene en función de la escolaridad y la experiencia. Para este fin relaciona el logaritmo de los ingresos, los años de educación (s) y la experiencia laboral $(\mathrm{x})^{4}$. «En los últimos 50 ańos, virtualmente todo trabajo empírico que necesitó una especificación para el ingreso laboral utilizó la denominada ecuación de Mincer» (Yamada, 2010, p.3).

$$
\operatorname{Ln} Y(s, x)=\beta_{0}+\beta_{1} s+\beta_{2} x+\beta_{3} x^{2}+\varepsilon
$$

El modelo econométrico de Jacob Mincer operacionaliza el concepto de capital humano, lo vuelve fácil de aplicar en las investigaciones concretas. Esta ecuación, así formulada, fue objeto de posteriores precisiones para los posibles sesgos que aparecen en su uso. La ecuación se focaliza más en la oferta de capital humano y prescinde de la demanda creada en el mercado de trabajo.

La Ecuación de Mincer halla varias tasas de rendimiento en el mercado educativo y laboral, lo cual

4 «La estimación del coeficiente asociado a los años de educación en una ecuación de ingresos laborales a la Mincer ha sido el enfoque tradicional para computar los retornos a la educación en el mercado laboral en casi todos los países del mundo. La validez de estos estimados, sin embargo, dependen de una serie de supuestos que determinan tanto el uso de ese coeficiente como el uso de la educación para caracterizar el perfil de ingresos para diferentes ańos de escolaridad y experiencia» (Yamada, 2010, p.3) 
es resultado de los distintos tipos de experiencia potencial, según lo establecido por la teoría del capital humano.

\subsection{La OCDE y el capital humano}

La Organización para la Cooperación y el Desarrollo Económicos (OCDE) es una institución de cooperación internacional, compuesta por 34 estados, cuyo objetivo es coordinar sus políticas económicas y sociales (OCDE, 2016). Es una institución que desarrolla actualmente un impulso singular en la difusión de la teoría del capital humano (Keeley, 2014). Ha desarrollado propuestas influyentes acerca de este punto, sobre todo, en el terreno de la educación.

Los estudiantes y los solicitantes de empleo en Perú necesitan más ayuda en su exploración de los cada vez más complejos mercados educativo y laboral del país, de modo que puedan realizar inversiones acertadas. [...] Seguir ampliando y mejorando [...] otras herramientas orientadas al consumidor que ayudan a aumentar la sensibilización sobre las tendencias y las oportunidades del mercado laboral, así como el coste y la rentabilidad de la inversión en relación con programas concretos. (2016, pp. 6-8)

La OCDE define el capital humano en los siguientes términos: "Los conocimientos, habilidades, competencias y atributos incorporados en los individuos y que facilitan la creación de bienestar personal, social y económico" (Keeley, 2014, p. 31).Considera que su producción es fundamental para el avance de los países menos desarrollados.

La OCDE ha ideado, entre otros mecanismos de evaluación, las famosas pruebas PISA,

\subsection{En resumen}

El capital humano queda definido como un conjunto de conocimientos, capacidades, habilidades, destrezas, salud, cuya adquisición eleva la capacidad productiva y los ingresos futuros. Schultz, Becker y Mincer expresan que estas características se adquieren por el estudio, por la formación y la experiencia. La educación ${ }^{5}$ queda definida así como una inversión para obtener capital humano.

5 Según la teoría del capital humano la educación es una inversión que realiza para incrementar su capacidad productiva futura» (Yamada, G y Cárdenas, M., 2007, p. 54). Para Villalobos (2009) la educación
La inversión adquiere muchas formas de manifestarse: escolarización, estudios de posgrado, formación en el empleo, atención médica, migraciones, adquisición de información sobre el mercado, otros aprendizajes.

La tasa de retorno resulta ser, en la teoría del capital humano, el resorte principal en el desenvolvimiento del «mercado de la educación». Gran impacto tendrá el curso que siga la oferta y la demanda, $y$, sobre todo, la perspectiva de crecimiento de esta última, hasta que su rendimiento remunerativo no decaiga a límites desalentadores para la inversión.

\section{Premisas del programa beca $\mathbf{1 8}$ como formador de capital humano}

El programa parte de las siguientes consideraciones:

1. Caracteriza al Perú como un país en vías de desarrollo, por lo cual considera que es fundamental para el Estado formular planes de desarrollo de capital humano a la vez que se logra fomentar la inclusión social (Pronabec, 2013, p. 15).

2. Es necesario lograr la participación de la población en situación de pobreza en la actividad económica y en la mejora de la productividad, pues es la más vulnerable al desempleo (Pronabec, 2013, p. 15).

3. La educación debe suponer la creación de capital humano, y la búsqueda de un mejor beneficio a futuro (Pronabec, 2013, p. 16).

4. Para el crecimiento económico se requiere un Sistema Nacional de Innovación con tres aspectos: primero, recursos humanos; segundo, infraestructura adecuada; tercero, instituciones que vinculen investigación con empresas productoras de bienes y servicios (Pronabec, 2013, p. 16).

5. El Estado, según este punto de vista, debe desarrollar capital humano, el cual debe estar «capacitado para integrar la producción industrial en el marco del desarrollo del país» (Pronabec, 2013, p. 16).

6. La estrategia que el país requiere para el desarrollo debe ser "Universidad - gobierno - industria» (Pronabec, 2013, p. 16) Este hecho fue plantea-

vinculada al desarrollo del capital humano implica el empleo de dicho capital para obtener un beneficio a futuro, calculado de acuerdo al rendimiento.( p.285) 
do desde la propuesta de la diversificación productiva, hoy abandonada.

7. En el área rural, donde está la mayor población indígena, solo 6,7\% alcanzó educación superior. Esta situación plantea el problema de cómo incluir estos grupos humanos en los planes de desarrollo mediante la inversión en educación (Pronabec, 2013, p. 17)

8. La creación de los programas de becas deben, entonces, orientarse al apoyo del desarrollo del capital humano, pues elimina la pobreza y crea mano de obra calificada necesaria al empresariado.

\subsection{El análisis del problema de la movilidad social hecho por el programa Beca 18 ha considerado las siguientes características}

a) Limitado acceso a estudios superiores. «El 15,7\% de la población peruana cuenta con estudios superiores completos, $8,5 \%$ corresponden a la educación superior técnica completa, 7,2\% a la educación superior universitaria completa. Alrededor del 80\% tiene una probabilidad superior al $10 \%$, de ser pobre en algún momento de su vida» (Pronabec, 2014, p. 21).

Pese al impacto que tiene el acceso y culminación de los estudios superiores en la reducción de la probabilidad de ser pobre monetario en el Perú, solo el 18\% de los jóvenes entre 20 y 27 ańos ha alcanzado este nivel, según la Encuesta Nacional de Hogares (2012).

b) A más educación superior, más productividad, más salario. Una persona con educación superior es cuatro o seis veces más productiva que una sin educación (Pronabec, 2012).Culminar los estudios de educación superior significa la posibilidad de obtener un salario entre cuatro o seis veces mayor.

c) El problema del retorno de la inversión en educación en el Perú. Los teóricos del capital humano en el Perú introdujeron en el análisis del problema la cuestión del «retorno", de cuan rentable resulta invertir en la educación frente a otras posibilidades de ganancia. Así:

un año más de educación primaria y secundaria incrementa el salario por hora en $5 \%$ y $7 \%$ respectivamente. No obstante, la educación superior universitaria y técnica muestra un retorno bastante mayor, especialmente cuando son instituciones privadas. En particular, un año más de educación superior en una universidad privada rinde $17 \%$, mientras que en un instituto privado $13 \%$ (Pronabec, 2014, p. 23).

d) Acceso a la educación superior, un sesgo antipobre. El número de universidades en el Perú se ha incrementado, especialmente el de las universidades privadas, igual sucede con el número de matriculados (ANR, 2013, pp.10-15). Los sectores económicos y sociales medios se esfuerzan por ingresar a estas instituciones y así elevar su estatus social y económico. «El número de jóvenes de edades entre los 17 y 25 años, matriculados en la educación superior, pasó de 848 mil 435 matriculados en el ańo 2002; a 1'171,590 en el 2012: un incremento de 38,09\%" (Pronabec, 2014, p.27). «Uno de cada diez matriculados en educación superior proviene de un hogar que vive en condición de pobreza; [...] el acceso a la educación superior está afectado por el nivel socioeconómico del hogar de procedencia» (Pronabec, 2014, p.28). Examinada la tasa de matrícula por quintiles, se observa que al quintil uno, el de más bajos ingresos, le corresponde el 7\% de las matrículas, en tanto que al quintil 5 , el de más altos ingresos, la tasa que le corresponde es del $42 \%$ (Pronabec, 2014, p.28).

Estas cifras relativas subrayan el sesgo antipobre que existe en la educación superior. Los hogares pobres carecen de recursos suficientes para superar las exigencias financieras, enfrentan así una barrera infranqueable.

Si bien es cierto que las restricciones financieras de corto plazo por parte de hogares pobres son un fuerte impedimento para que sus hijos puedan acceder a la educación superior, también se deben considerar las restricciones de largo plazo, las cuales no son necesariamente monetarias (Pronabec, 2014, p. 26).

e) Más universidades, más matrículas, menos calidad en la educación superior. El incremento de la matrícula en la educación superior en el Perú no ha conducido a una mejora en la calidad de la misma. Mientras que la tasa neta de matrícula 
tiene un incremento relativo cercano a 10 puntos en estos últimos años, la tasa de culminación se mantiene en un aproximado 17\% (Pronabec, 2014, p. 31). Pero, como señala Pronabec: «Estas tasas de matrícula y culminación tienen implícita una tasa de deserción, la cual se encuentra por encima del 30\% (Yamada, Castro y Rivera 2012)» (PRONABEC, 2014, p. 31).

f) Tasa de subempleo de egresados universitarios. El crecimiento del número de egresados de universidades e institutos de educación superior, así como el perfil de estos profesionales formados, no guardan correspondencia con las características de número y calidad que demandan las empresas y negocios del país. Muchos alumnos de bajo nivel académico ingresan a universidades de cuestionable calidad y devienen en candidatos seguros al desempleo. Estas instituciones de servicios educativos, aseguran la ganancia y propician el incremento del número de matrículas, a costa de ofrecer un servicio de mala calidad y de poco valor en el mercado de trabajo. «El 35\% de egresados universitarios en el ańo 2012 no trabajaban en una ocupación para la cual se prepararon [...] las tasas de empleo no han crecido en el mismo nivel que la población de egresados de la educación superior» (PRONABEC, 2014, p.31).

\section{El programa Beca 18}

Frente a una situación como la descrita, el gobierno diseña este programa como parte de sus políticas sociales, y como una forma concreta de implementación de la política de inclusión social. El propósito del programa Beca 18 es dotar de una carrera a una parte de este sector social en condición de pobreza y pobreza extrema. El programa infiere que, logrado este objetivo, los beneficiados estarán en posibilidad de obtener un sueldo que será superior a la línea de pobreza.

Basándose en las tesis de la teoría del capital humano y en los más recientes aportes de la OCDE sobre el punto, consideran fundamental el apoyo económico y académico de parte del Estado.

La población potencial a la que va dirigido el programa Beca 18 es definida como "población en proceso de inclusión a la educación superior». Tiene propuesto atender, inicialmente, cinco mil jóvenes en el año 2012 y 25 mil hasta el 2016, metas que pueden ser ampliamente superadas.

En el ámbito interno, Beca 18 cubre los costos de nivelación, tutoría, inscripción, matrícula, pensión, materiales de estudio, idioma, titulación; así como gastos de alimentación, transporte y seguro médico; alojamiento, siempre que los beneficiarios residan en un lugar distinto al de su centro de estudios (MED, 2011, 2012, 2014).

Los jóvenes tienen que haber ingresado a la universidad, no haber iniciado sus estudios, ser de situación de pobreza o pobreza extrema, pertenecer a las carreras de ciencias básicas, tecnológicas y otras seleccionadas; aceptar el compromiso de trabajar durante tres años después de titulados en su localidad, de preferencia. «Las carreras profesionales que serán elegibles deberán ser aquellas con mayor retorno en el mercado laboral y enmarcarse dentro de las áreas [...] priorizadas por el Plan Nacional de CTI para la Competitividad y el Desarrollo Humano 20062021» (MED, 2012, p. 4).

\section{Discusión de resultados}

La lucha que sostienen los peruanos por acceder a la educación de calidad, la ciencia y a la alta tecnología es el esfuerzo por la democratización de la enseñanza y porque se respete la pluriculturalidad en ella. Esta exigencia tiene como resultantes la formulación de programas gubernamentales como el caso de Beca 18 .

Este programa, al definir la educación como una inversión efectuada para incrementar la capacidad productiva futura (Yamada y Cárdenas, 2007, p. 54), la ubica dentro de los parámetros que corresponden a las operaciones financieras, y son estos criterios los que se utilizan para juzgar su viabilidad e inviabilidad, su éxito o fracaso y la naturaleza del apoyo que ofrece. Así, la rentabilidad de la operación siempre está presente en las reflexiones y decisiones que se adoptan; por eso, al seleccionar el sector social al que se dirige, escoge un grupo muy selecto de alumnos en condición de pobreza o pobreza extrema, que cumplen ciertas exigencias y que fácilmente podrían forjarlo como fuerza de trabajo calificada, puesta a inmediata disposición del mercado laboral. En esta misma dirección, al relacionar eficiencia y equidad, sostiene: 
En este tipo de programas, el balance entre eficiencia y equidad será difícil de lograr: si en aras de la equidad buscamos transferir recursos a los más pobres corremos un mayor riesgo de estar interviniendo sobre una población donde las restricciones en términos de habilidades son más fuertes y, por lo mismo, corremos un mayor riesgo de que estos recursos no sean bien aprovechados (es más probable que el joven termine desertando o, si se gradúa, sea de una institución de poca calidad) (Pronabec, 2013, p. 3).

De aquí se entiende que el sesgo antipobre ${ }^{6}$ de la educación superior debe ser entendido, no solo como un problema ocasionado por la escasez de recursos monetarios, sino también de conocimientos, habilidades y capacidades no desarrollados.

Así, la teoría del capital humano y algunas investigaciones como las de Carneiro y Heckman (2002), señalan que las becas para seguir estudios en el nivel superior, dirigidas a jóvenes de estos sectores sociales, deben tomar en cuenta dos tipos de restricciones: el primer tipo, las de corto plazo, de naturaleza financiera, que tiene que ver con la falta de recursos económicos; el segundo, de largo plazo, y que corresponde a la mala calidad de la instrucción básica que han alcanzado estos jóvenes y a las limitaciones académicas y culturales del entorno familiar. Las limitaciones del segundo tipo son las más difíciles de superar, y son las que motivan en los teóricos del capital humano políticas más severas y excluyentes. Por eso, del sector social en condición de pobreza y pobreza extrema solo se brinda apoyo a los jóvenes que posibilitan una mejor articulación entre educación básica y superior. El programa solo acoge a los menos afectados por la pobreza, a aquellos que sobresalgan por el desarrollo de sus habilidades cognitivas y conocimientos. La mejora de los conocimientos recibidos en el nivel básico escolar, está reducida al ciclo de nivelación u otras formas menores de cubrir los vacíos y salvar deficiencias. Incrementarlos afectaría la rentabilidad del programa.

6 Los quintiles de menores ingresos (con mayor probabilidad de pobreza) tienen menor porcentaje de ingreso a la educación superior; en cambio, los quintiles superiores (de mayores ingresos) tienen mayor porcentaje de ingreso a la educación superior (Pronabec, 2014, p.28).
Los teóricos del capital humano no tienen muchas expectativas en mejorar el nivel de la formación básica de los ganadores de beca.

Los jóvenes que no logren acceder al programa pasan a ser el costo social del sistema económico y político existente.

El programa en sí resulta muy focalizado por los partidarios de la teoría de capital humano, pues sostienen que:

No debemos sobreestimar el impacto de un esquema de becas y/o créditos focalizado: parte importante de los problemas de acceso de las familias pobres es el resultado de restricciones de largo plazo que no pueden ser revertidas con una transferencia corriente de dinero (Castro, 2011, p. 3).

Este criterio es incluido textualmente en el documento Programa Nacional Beca 18 «Plan de Gestión Institucional 2012».

Los partidarios de la teoría del capital humano concluyen que «es indiscutible la necesidad de que cualquier esquema de becas y/o crédito focalizado en jóvenes de escasos recursos económicos venga también acompañado de un riguroso mecanismo de selección de beneficiarios que esté basado en su potencial académico" (Castro, 2011, p. 3).

La «inclusión social» de la que habla el programa tiene estos fines y límites que brotan de su concepción misma, de su fundamento teórico. La privación de conocimientos, capacidades y habilidades generadas por el sistema socioeconómico termina siendo más restrictiva que la pobreza monetaria.

Los pobres que no alcancen este nivel mínimo exigido se pierden para la humanidad.

En la implementación de este programa, surgen, entonces, dos conflictos importantes:

El primero, entre la rentabilidad que busca el Estado versus los mayores recursos necesarios para superar las limitaciones académicas de la educación básica de los becarios y becarias. Por eso, debido al creciente número de jóvenes que solicitan la beca, y debido a los escasos recursos económicos que destina el Estado, se introduce la necesidad de realizar una rigurosa focalización, ajustada al criterio de rentabilidad de la inversión y no al de la educación como derecho humano. La dirección del programa Beca 18 ha decidido, en este sentido, resolver las dificultades con 
una mayor elitización, lo cual resulta incongruente con la política de inclusión y democratización.

El segundo conflicto es el que existe entre la pobreza de la infraestructura productiva del país versus las mejores condiciones materiales de producción que requiere la formación de profesionales avanzados. La dirección del programa Beca 18 y el Estado han decidido, en este sentido, inclinarse hacia la formación de mano de obra calificada en un nivel que no desborda las necesidades del sistema productivo.

De estas dos consideraciones se llega a establecer los siguientes puntos:

a. El aspecto central en el programa es considerar la educación como inversión rentable y generadora de capital humano, y no así respetar la educación como un derecho humano.

b. El programa se plantea como objetivo clave elevar el ingreso futuro de los becarios y permitirles, así, salir de la situación de pobreza monetaria en que se hallan. A este fin subordina su intervención.

c. El programa pone el requisito de preservar el retorno de la inversión para que resulte viable.

d. Las carreras que principalmente apoyan son las de elevada rentabilidad, sobre todo las tecnológicas y técnicas, y desarrolla, directa e indirectamente, un desinterés por las humanidades y las ciencias sociales.

El problema del origen de la pobreza. La teoría del capital humano hace recaer la responsabilidad de la desigualdad social en los mismos trabajadores, y no en el sistema capitalista. Si los trabajadores quisieran de verdad mejorar su condición económica y salir de la situación de pobreza, nos dice, el camino sería invertir, correctamente, más en educación y, de esta manera, lograr una elevada formación técnica y profesional. Todos serían capitalistas (Schultz, 1999). El resultado de esta decisión sería una mejora en cuanto a su productividad y una mejora de sus posibilidades de competencia en el mercado de trabajo. Por tanto, según esta postura, la solución a la pobreza no está ligada a la naturaleza del sistema, sino a la decisión del propio trabajador. Eluden, de esta forma, la relación histórica, causal, que existe entre sistema capitalista y pobreza, postura que es ya característica de la teoría del capital humano. Marx liga la pobreza a su correspondiente modo de producción:
El proceso capitalista de producción reproduce (...) en virtud de su propio desarrollo, el divorcio entre la fuerza de trabajo y las condiciones de trabajo. Reproduce y eterniza, con ellos, las condiciones de explotación del obrero. Le obliga constantemente a vender su fuerza de trabajo para poder vivir y permite constantemente al capitalista comprársela para enriquecerse (...) por tanto, el proceso capitalista de producción, enfocado en conjunto o como proceso de reproducción, no produce solamente mercancías, no produce solamente plusvalía sino que produce y reproduce el mismo régimen del capital: de una parte al capitalista y de la otra al obrero asalariado (Marx, 1999, pp. 486-487).

«El capital presupone el trabajo asalariado y este el capital. Ambos se condicionan recíprocamente y se crean el uno al otro" (Marx, 1999, p. 487, nota 21). La riqueza de uno se logra a costa de la pobreza del otro, situación reproducida cotidianamente. El capital lleva la exigencia de "reducir los costos laborales» (Eliminar o reducir vacaciones, reducir el salario real, eliminar seguro de salud, eliminar jubilación y otros).

Aquí, nadie compra la fuerza de trabajo para satisfacer, con sus servicios o su producto las necesidades personales del comprador. No, la finalidad de este acto es explotar el capital, producir mercancías, que encierran más trabajo del que paga el que se las apropia y aquí, por tanto, contienen una parte de valor que al capitalista no le cuesta nada y que, sin embargo, puede realizarse mediante la venta de las mercancías. La producción de plusvalía, la obtención de lucro; tal es la ley absoluta de este sistema de producción (Marx, 1999, p. 522).

La teoría Schultz y Becker al reducir la pobreza a un problema de ingreso monetario, de distribución; al no ubicarla en su estrecha relación con el modo de producción que la determina; al atribuirla a la sola falta de inversión en educación, sin advertir su origen estructural, termina por llevar la explicación a la situación de un simple desajuste de la oferta y la demanda, a entenderla como un asunto de alta o baja cotización. Reduce así al trabajador a la posición antihumanista de tener que lograr cotizarse mejor como una mercancía: lleva a la cosificación del ser humano.

Amartya Sen, con mejor criterio, señala que la pobreza «debe concebirse como la privación de ca- 
pacidades básicas y no meramente como la falta de ingresos» (Sen, 2000, p.114). "privación» que no es resultado de un "descuido" personal.

La adquisición de conocimientos, competencias, capacidades y actitudes positivas ofrece mayores $\mathrm{y}$ mejores posibilidades de empleo e ingreso económico; posiciona mejor al trabajador en el mercado de trabajo, hecho ya considerado por el propio Adam Smith (1983, pp. 152-153), pero programas de subvención como Beca 18 , solo posibilitan el acceso a un número restringido de jóvenes estudiantes en situación de pobreza, la gran masa permanece aún sin hallar una solución. Queda así en pie el problema de ¿Cómo democratizar la educación de buena calidad? ¿Cómo eliminamos este tipo de pobreza que también genera el sistema capitalista?

¿El problema es mejorar los criterios de elección de una carrera, incrementar la competencia y aportar más información del mercado? Para la teoría del capital humano el esfuerzo debe ser orientado a mejorar el mercado educativo. Sin embargo, la libertad para elegir y acceder a una carrera, tal como lo sostiene el capitalismo, termina convertida en una clara ficción. Varios obstáculos se oponen a ello.

El primer gran obstáculo que enfrenta es la desigualdad económica, social y cultural de la población. Por eso, incrementar la competencia entre instituciones educativas no resuelve el problema planteado a los sectores en situación de pobreza y pobreza extrema.

Un segundo obstáculo es la existencia de una economía primario exportadora y el proceso de reprimarización de la economía, verdaderos obstáculos al desarrollo del país, pues contribuyen a preservar y reforzar los viejos males de la educación en el Perú.

El estado, a su vez, reduce su función a conformar la enseñanza con las necesidades precarias del empresariado.

Por estas razones, el sesgo antipobre de la educación superior no es posible eliminarlo con una mejor información sobre los rankings de las instituciones educativas o sobre los buenos sueldos de sus egresados. Los teóricos del capital humano usan, estos medios con el fin de "crear mercado educativo", de guiar postulantes hacia ciertas carreras e instituciones que demanda el empresariado, y, finalmente, bajar su valor. Piensan que el problema es impulsar más políticas de mercado; van en busca de esa gran masa de la clase media que, con mucho esfuerzo y grandes limitaciones, demanda educación superior. Pero, aun en estos casos, resulta ineficaz por tratarse de un conocimiento impreciso sobre la situación del mercado laboral de cada una de las carreras, por ser una débil proyección para un quinquenio. Esta política resulta extrańa a la perspectiva de desarrollo de un Proyecto Nacional.

La anarquía de la producción, propia del sistema capitalista, constituye otra gran dificultad para planificar el desarrollo educativo, situación agravada en el Perú con más de veinticinco años de aplicación de las políticas del Consenso de Washington.

Por otro lado, el factor vocación, cultivado desde la educación básica, y reforzado en el ámbito familiar, es el que mantiene una influencia decisiva al elegir una carrera. La vocación es sólida en algunos casos, y débil en otros; la razón es que no todos cuentan con las competencias, conocimientos y habilidades propias de la carrera que desean seguir.

La teoría del capital humano califica de romántica y poco empresarial a esta cultura de elegir principalmente por vocación; en contraposición, alienta una decisión más utilitaria y pragmática en los jóvenes; los convoca a decidir en función de la ganancia monetaria y a omitir la visión social. Alienta el abandono de la vocación, si se avizora un menor ingreso económico. Alienta, además, el recelo y rechazo contra las humanidades (Nussbaum, 2010).

Sobre la determinación de las diferencias salariales. Una de las principales tesis de la teoría del capital humano afirma que las diferencias de cualificación de la fuerza de trabajo determinan las diferencias de los salarios obtenidos. A partir de esta deducción unilateral y mecanicista, consideran que la mayor remuneración debe ser el factor decisivo para la elección de una carrera o grado de estudio.

Sobre este punto, el valor de la fuerza de trabajo, son muy importantes las tesis planteadas por Karl Marx:

Para modificar la naturaleza humana corriente y desarrollar la habilidad y la destreza de hombre para un trabajo determinado, desarrollando y especializando su fuerza de trabajo, hácese necesaria una determinada cultura o instrucción, que, a su vez, exige una suma mayor o menor de equivalentes de mercancías. Los gastos de educación de la fuerza de trabajo varían según el carácter más o menos calificado de ésta. Por tanto, estos gastos de 
aprendizaje, que son insignificantes tratándose de la fuerza de trabajo corriente, entran en la suma de los valores invertidos en su producción.

El valor de la fuerza de trabajo se reduce al valor de una determinada suma de medios de vida. Cambia, por tanto, al cambiar el valor de éstos, es decir, al aumentar o disminuir el tiempo de trabajo necesario para su producción (1999, p. 125).

El trabajo considerado como trabajo más complejo, más elevado que el trabajo social medio, es la manifestación de una fuerza de trabajo que representa gastos de preparación superiores a los normales cuya producción representa más tiempo de trabajo y, por tanto un valor superior al de la fuerza de trabajo simple. Esta fuerza de trabajo de valor superior al normal se traduce, como es lógico, en un trabajo superior, materializándose, por tanto, durante los mismos períodos de tiempo, en valores relativamente más altos (1999, p. 148).

A una mayor cualificación de la fuerza de trabajo, tal como lo precisa Marx, correspondería un mayor valor, dado que el tiempo de trabajo socialmente necesario para producirla deberá incrementarse también. El valor muestra así su carácter dinámico, histórico y aparece como un punto de oscilación de los salarios. Esto constituye una tendencia que opera en el largo plazo y bajo la forma de constantes desviaciones de este valor medio. No como algo que se cumple de manera rígida. Determinado el valor socialmente necesario para producir y reproducir la fuerza de trabajo, factores como las características del mercado, la oferta y la demanda, el ejército industrial de reserva, el nivel de organización sindical y lucha de los trabajadores, las relaciones sociales (capital social), los ciclos del capital, todos juegan en este mundo de oscilaciones, ocasionando las alzas y bajas, en relación al valor medio. Clave aquí es el tipo de relaciones de producción y reproducción que se expresan en la fuerza de trabajo.

Otro factor que lleva hacia abajo o hacia arriba los salarios es el nivel tecnológico de las empresas, aquí importa mucho la calidad y la cantidad invertida en máquinas, equipos (el capital constante), en su nivel de innovación; asimismo la inversión destinada a incorporar fuerza de trabajo. Esta relación proporcional, histórica y dinámica, es lo que Marx llama la composición orgánica de capital. Por esta razón, es unilateral considerar la preparación del trabajador como el único elemento clave de la productividad, del crecimiento económico y de la distribución del valor producido, ignorando la naturaleza del proceso de acumulación del capital, los mecanismos y el funcionamiento de la sociedad capitalista. La fuerza de trabajo opera en una infraestructura productiva concreta, que puede ser avanzada, y entonces eleva su calidad; o atrasada, y entonces se mediocriza.

Es importante considerar que, en épocas de depresión y crisis, los despidos se incrementan y el salario real se reduce; resultan igualmente nefastos los llamados reajustes de las empresas. En el Perú un ejemplo claro lo tenemos en la política laboral del Consenso de Washington, implementada desde los noventa por el gobierno de Alberto Fujimori, la cual ha llevado a la precarización del empleo, de los sueldos y salarios. En períodos de aumento de la tasa de la ganancia, los salarios pueden aumentar, pero esto queda sujeto a la correlación de fuerzas y lucha organizada que se libre, según la lucha sindical y política que se realice. Está el ejemplo del magisterio con el SUTEP. Otro caso es el conjunto de criterios que observa el Consejo Nacional del Trabajo para incrementar el salario mínimo vital. El capitalismo siempre ha tendido a incrementar la ganancia, y por tanto, la plusvalía, y a reducir el valor del trabajo necesario. No es, entonces, mecánica y simplista la relación entre estudios y sueldos como la plantea la teoría del capital humano.

Bourdieu (2001), desde su punto de vista sociológico, subraya el hecho de que en la teoría del capital humano el rendimiento de la inversión escolar solo ve las inversiones y beneficios monetarios; critica la desatención a la inversión educativa socialmente más eficaz representada por la transmisión de capital cultural en el seno de la familia; critica que ignore que el «talento» y la «capacidad» son resultado de tiempo y de capital cultural; critica que se pase por alto el hecho de que el rendimiento escolar de la acción educativa depende del capital cultural previamente aportado por la familia, y que olvide que el rendimiento social y económico de los títulos y grados depende del capital social heredado que se puede movilizar en su respaldo (Bourdieu, 2001, pp. 137-138).

Estudio y tasa de retorno. Un joven egresado de un centro de educación superior, apoyado por el programa Beca 18, está en mejores posibilidades de empleo 
frente a los que no lograron acceder a estudios superiores. No cabe duda que el apoyo recibido resultará importante en la vida presente y futura del estudiante. Esto no ofrece duda, sino no se entendería la creciente demanda que tiene el programa. Sin embargo, el sector social que está fuera del programa es mucho más numeroso que el beneficiado. Las mejoras restringidas e individuales no bastan para afirmar que el programa Beca 18 opera un cambio estratégico en la fuerza laboral del país o que el sistema capitalista no es responsable de la pobreza. Como vemos, las distancias entre los niveles de ingreso dependerán de muchas otras condiciones ya señaladas.

\section{Conclusiones}

- Beca 18 es uno de los programas emblemáticos de la política social del gobierno de Ollanta Humala. Ha suscitado gran expectativa en la población juvenil de menos recursos económicos, como un medio de acceso a la educación superior.

- Considera a la educación como una inversión y como factor determinante de los ingresos individuales. Las metas del programa, sus expectativas e indicadores, los filtros que ha establecido, confirman a la teoría del capital humano como su fundamento.

- Beca 18 exige una "inversión rentable» para el Estado. Por eso reduce los fondos destinados a subsanar las limitaciones de la educación básica, factor de mayor inestabilidad para los becarios.

- La teoría del capital humano sesga la oferta de carreras hacia el ámbito tecnológico, de gran rentabilidad, desarrollando el prejuicio contra las carreras de humanidades y de las ciencias sociales. Asume, así, una posición utilitaria y pragmática.

- Al no lograr ubicar la pobreza en su estrecha relación con el modo de producción que la determina, la reduce a un problema de ingreso monetario, de distribución, de una simple mejora de la oferta y la demanda,

- El programa se contradice con la gran informalidad existente en el Perú y con una pobre estructura productiva, primario exportadora, altamente monopolizada, que no constituye aliento para el desarrollo científico y tecnológico.

\section{Referencias bibliográficas}

Asamblea Nacional de Rectores (2013). Tips de universidades. Lima: ANR.

Becker, G. (1983). El capital humano. Madrid: .Alianza Universidad de Textos, Alianza Editorial, S.A.

Bourdieu, P. (2001). Poder, derecho y clases sociales. Bilbao: Editorial DESCLÉE de Brouwer.

Carneiro, P. y Heckman, J. (2002). La política del Capital Humano. Trabajo presentado en el Seminario Alvin Hansen en la Universidad de Harvard el 25 de abril de 2002106 páginas

Castro, J., Casavilca, P., Lizarzaburu, R. (2010) Crecimiento económico y demanda por educación superior en el Perú: un estudio para el período 20042006. En: Apuntes 66. Centro de investigación de la Universidad del Pacífico.

Castro, J. (2011). ¿Por qué los pobres no van a la universidad? En: SABERES COMPARTIDOS, www.saberescompartidos.pe

INEI (2012). Informe técnico Evolución de la pobreza 20072011. Lima: INEI.

Keeley, B. (2014). Capital humano. Cómo influye en su vida lo que usted sabe. España: Castillo. Biblioteca Integral. Percepciones de la OCDE.

MarX, K. (1999 [1867]). El capital T. I. Traducción: Wenceslao Roces (3ra. Ed.). México: Fondo de Cultura Económica.

Ministerio de Educación (2011). Propuesta inicial Beca 18. Lima: Ministerio de Educación. Disponible en: http://www.minedu.gob.pe/obec/beca18.php

Ministerio de EduCaCión (2012). Resolución Ministerial Nro.0080- 2012-ED

Ministerio de Educación (2014). Lineamientos de Política para un sistema de Becas, Créditos Subsidiados por una Educación Superior de Calidad.

Nussbaum, M. (2010). Sin fines de lucro. Por qué la democracia necesita de las humanidades. Buenos Aires: Katz ediciones.

Ordine, N. (2013). La utilidad de lo inútil. Barcelona: Acantilado.

Pronabec (2013). Memoria Anual 2013

Pronabec (2014). Memoria Anual 2014

Rivera, M., Castro, J., y Yamada, G. (2012). Educación superior para el Perú: retos para el aseguramiento de la calidad. Estudio encargado por SINEACE. 
Schultz, T.W. (1999). La inversión en capital humano. En Mariano F. Enguita (editor) Sociología de la educación. Espańa: Editorial Ariel.

Sen, A. (2000). Desarrollo y libertad. Argentina: Editorial Planeta.

Sмiтh, A. (1983 [1776]). La riqueza de las naciones. T. I Barcelona: Ediciones Orbis sa. Libro I. Biblioteca de Economía.

Villalobos, G. y Pedroza, R. (2009). «Perspectivas de la teoría del capital humano acerca de la relación entre educación y desarrollo económico». Tiempo de Educar 10(20): 273: 306.

YAMADA, G. (2007a). Educación Superior en el Perú: Rentabilidad incierta y poco conocida En: Economía y sociedad 63, CIES, pp. 53-61.
YAMADA, G. (2007b). Retornos a la educación laboral en el mercado: ¿Vale la pena el esfuerzo? Lima: Universidad del Pacífico: Documento de trabajo 78

Yamada, G. y Castro, J. (2010). Educación superior e ingresos laborales: estimaciones paramétricas y no paramétricas de la rentabilidad de la rentabilidad por niveles y carreras en el Perú.

Yamada, G., Lavado, P. y Oviedo, N. (2016). Educación superior y subempleo profesional: ¿una creciente burbuja mundial? DD 1609 Universidad del Pacífico Lima: CIUP. 